\section{Grand unification magnetic monopoles inside the Earth}

CARRIGAN $^{1}$ has suggested that the equilibrium between the magnetic and gravitational forces would lead to these monopoles being trapped in two positions on the magnetic axis, one each side of the centre, depending on their polarity. However, his discussion is incorrect in several respects. First, in the Earth's core, the gravitational field is roughly proportional to radius $r$. If the magnetic field were due to a central dipole it would be proportional to $r^{-3}$, and there would be (radial) equilibrium on the axis at $\sim r=$ $0.18 \boldsymbol{R}_{e}$, within the fluid core, as he states. But the magnetic field is far from being dipolar, and within the core the dipole and non-dipole fields are comparable in magnitude; even at the core surface there are already $\sim 14$ positions ${ }^{2}$ where the field is radial, not just the two he assumed.

Second, in fact, the magnetic field is produced by electric currents in the core; reasonable current distributions give a dipole radial component of field which increases only very slowly with depth. This would give an 'equilibrium' radius of $\sim 0.007 \boldsymbol{R}_{e}$, only $50 \mathrm{~km}$ from the centre, and well within the solid inner core. Third, his 'equilibrium' calculations have apparently considered only radial forces, and for his dipole 'equilibrium' the monopole is unstable to infinitesimal tangential displacements. Stable equilibrium would require complicated fields with a most unlikely coincidence of magnitude and topology.

Finally, as Carrigan points out, in the core there are probably toroidal fields much larger than the poloidal dipole fields. However, these toroidal fields have no radial component, and will be largely latitudinal, so will not "result in large axial fields". What they will do is make it even less likely that there will be any stable positions of purely radial field. The inner core also will have large toroidal fields ${ }^{3}$.

It does seem most unlikely that there will be any significant trapping of monopoles by the mechanism Carrigan suggests.

\section{F. J. LOWES}

School of Physics, The University, Newcastle upon Tyne, NE1 7RU, UK

1. Carrigan, R. A. Jr Nature 288, 348-350 (1980).

2. Barraclough, D. R. \& Malin, S. R. C. Geophys. J.R. astr. Soc. 65, 467-473 (1981)

3. Gubbins, D. J. geophys. Res. (in the press).

CARRIGAN REPLIES-Because of the limitations of space I could not emphasize the model nature of the calculation. However, I did point out that the dipole model was not a proper picture of the interior of the Earth. I did not say a monopole of one polarity would occupy a single region of equilibrium but rather that GUMMs of one polarity would interchange with those of the other polarity. I did not touch on one aspect of the situation, the fact that GUMMs would probably not move rapidly through solid material in the interior of the Earth. If this is so it represents a third effective force. Static or dynamic equilibrium would be a result of all three effective forces.

In view of the limits of the picturelittle is known about GUMM energy loss processes, there is incomplete knowledge of the interior of the Earth and little understanding of the accretion mechanism-it did not seem appropriate to develop a more comprehensive picture. The main point I wished to make was that even a relatively few GUMMs of opposite polarities could contribute a spectacular amount of energy.

\section{RICHARD A. CARRIGAN JR}

Fermi National Accelerator Laboratory, PO Box 500, Batavia,

Illinois 60510, USA

\section{Estimated speed of a giant bipedal dinosaur}

FROM observations of locomotion in diverse living animals, including ostrich, bird, man, horse and elephant, Alexander ${ }^{1}$ obtained a relationship between speed, stride length $(\lambda)$ and body size (expressed by $h$, height at the hip). This relationship seems to hold true, at least in general terms, for large and small animals, both bipeds and quadrupeds, at gaits from walk $(\lambda / h<2.0)$ to run $(\lambda / h>2.0)$. To estimate the speeds of certain dinosaurs Alexander applied this relationship to their tracks, where $\lambda$ could be measured directly and $h$ could be estimated from the size of the footprints. He found ${ }^{1}$ that the estimated speeds were rather lowbetween 1.0 and $3.6 \mathrm{~m} \mathrm{~s}^{-1}$ (3.6 and 13.1 $\mathrm{km} \mathrm{h}^{-1}$ ).

Alexander's method has since been applied to various other dinosaur tracks $^{2-5}$, but few of these have yielded speed estimates greater than about $4.2 \mathrm{~m}$ $\mathrm{s}^{-1}\left(15.0 \mathrm{~km} \mathrm{~h}^{-1}\right)$. A notable exception was provided by Russell and Béland ${ }^{2}$, who calculated that a short section of trackway in the Mesaverde Formation (Cretaceous) of Colorado ${ }^{6}$ had been made by a dinosaur running at a speed of $7.54 \mathrm{~m} \mathrm{~s}^{-1}$ $\left(27.1 \mathrm{~km} \mathrm{~h}^{-1}\right)$. The Colorado dinosaur was apparently a giant bipedal ornithischian (probably an ornithopod of the family Hadrosauridae), with $h$ being $\sim 3.44 \mathrm{~m}$ and a live body weight of $\sim 11$ tonnes ${ }^{2}$. Russell and Béland based their calculations on data published by Brown ${ }^{6}$, whose account of the Colorado trackway is rather ambiguous; in one place Brown referred ${ }^{6}$ to a "fifteen foot stride", while in another he mentioned that the dinosaur "stepped 15 feet 2 inches". A stride is commonly understood to comprise two consecutive steps, or paces ${ }^{7}$, though the terms 'stride' and 'step' have sometimes been used synonymously. Evidently Brown was referring to a step (or pace), for the distance of 15 feet was measured between two footprints which he identified as those of left and right feet. Russell and Béland apparently followed Brown's interpretation: they presumably took the figure of 15 feet to represent a step (left footprint to right footprint) and doubled it to obtain the figure of $9.25 \mathrm{~m}$ which they cite for stride length ${ }^{2}$.

I have examined photographs of the Colorado footprints (supplied by Dr E. S. Gaffney), and I suspect that Brown's interpretation of them may be wrong (see Fig. 1). The two footprints identified by Brown as left and right are very similar,

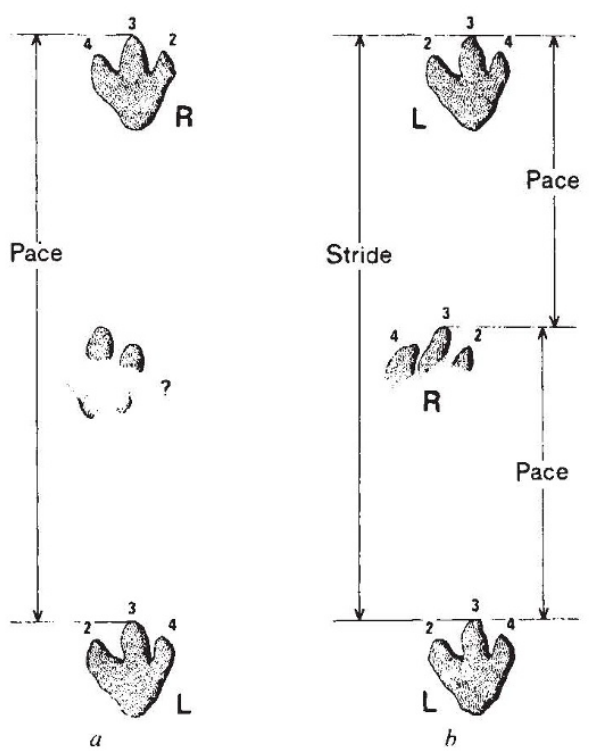

Fig. 1 Diagram illustrating two interpretations of ornithopod dinosaur trackway from the Cretaceous of Colorado (American Museum of Natural History, no. 3650). Each footprint has the digits numbered and is identified as left (L) or right (R). The footprints are represented by natural casts on the under-surface of a sandstone bed, so that left and right appear transposed. $a$, Brown's interpretation ${ }^{6}$, indicating a pace of $\sim 15$ feet $(4.57 \mathrm{~m})$; the surface feature marked ? is not regarded as part of the trackway. $b$, Revised interpretation, indicating a pace of $\sim 7.5$ feet $(2.29 \mathrm{~m})$ 